\title{
TRADUÇÃO ETNOGRÁFICA: A VIAGEM E SUA ESCRITA
}

\author{
Katia Aily Franco de Camargo ${ }^{1}$ \\ -Universidade Federal do Rio Grande do Norte, Natal, Rio Grande do Norte, Brasil
}

\begin{abstract}
Resumo: O presente artigo tem por objetivo apresentar uma proposta de análise do papel da linguagem/cultura na construção da(s) identidade(s) de um autor-viajante e, também, da identidade do outro. Acredita-se que tal proposta possa ser aplicada à literatura de viajante de uma maneira geral. De caráter eminentemente bibliográfico, tal proposta está baseada na descrição etnográfica enquanto realidade social apreendida a partir do "ver" (Laplantine, La description 1-128; Ferreira, Tradução etnográfica 55-91) e na relação desta com a tradução; nas três categorias de tradução propostas por Jakobson, a saber, intralingual, interlingual e inter-semiótica (63-72); e nas categorias de classificação de itens culturalmente marcados (ICE) elaboradas por Javier Franco (52-78). Como resultado, espera-se que tal proposta seja capaz de identificar as influências na construção da identidade do viajante e de seu "semelhante" estrangeiro.

Palavras-Chave: Tradução Etnográfica; Autor-Viajante; Relato de Viagem; Identidades
\end{abstract}

\section{ETHNOGRAPHYC TRANSLATION: THE TRAVEL AND THE WRINTING}

\begin{abstract}
This article aims to present a proposal of analysis: the role of language / culture in the construction of the travel writing identity as well as the identity of the other. We believed that such a proposal can be applied to travel-writings in general. Such proposal is eminently bibliographic and it's based on ethnographic description as a social reality apprehended from the "see" (Laplantine, La description 1-128; Ferreira, Tradução etnográfica 55-91) and its relationship with translation; in the three categories of translation proposed by Jakobson (63-72); namely intralingual, interlingual
\end{abstract}


and inter-semiotic; and in the classification categories of Cultural SpecificItem elaborated by Javier Franco (52-78). As a result, it is hoped that such a proposal will be able to identify the influences on the identity construction of the traveler and of his "foreign" fellow.

Keywords: Ethnographic Translation; Author-Traveller; Travel-Writing; Identities

Il faut tâcher de pénétrer ce qu'ils pensent, et non prétendre les faire penser à notre manière.

(Gerondo apud Laplantine 499)

\section{Introdução}

O presente artigo tem por objetivo apresentar uma proposta de análise do papel da linguagem/cultura na construção da(s) identidade(s) de um autor-viajante e, também, da identidade do outro. Tal proposta está baseada: na descrição etnográfica enquanto realidade social apreendida a partir do "ver" (Laplantine, La description 1-128; Ferreira, Tradução etnográfica 55-91) e na relação desta com a tradução; nas três categorias de tradução propostas por Jakobson (63-72); e nas categorias de classificação de itens culturalmente marcados (ICE) elaboradas por Javier Franco (52-78). Apesar de tratar-se de uma proposta, permito-me, à guisa de exemplo, pincelar, aqui e ali, breves análises de trechos de obras por meio das quais procurei mostrar essas identidades cambiantes de si e do outro.

Em 2016, é lançado, sob a direção de Denis Villeneuve, The Arrival, baseado no conto Story of your life do escritor estadunidense Ted Chiang. O filme de ficção científica, estrelado por Amy Adams, conta a história da chegada de seres interplanetários na Terra. A Dra . Louise Banks (A. Adams), uma linguista, é procurada por militares para traduzir os sons produzidos pelos alienígenas, pois era imprescindível saber se eles eram uma ameaça ou não para o planeta. In loco, Louise consegue, adotando um método visual rudimentar (quadro branco e pincel), decodificar o Heptapod, nome dado ao novo idioma. Questionada pelos militares a respeito 
de seu método tradutório/comunicativo, ela o explica escrevendo a seguinte frase numa lousa ${ }^{1}$ :

\section{-What is your purpose on Earth?}

Louise -First, we need to make sure that they understand what a question is. Okay? The nature of a request for information along with a response. Then, we need to clarify the difference between a specific "you" and a collective "you", because we don't want to know why Joe Alien is here. We want to know why they all landed. And 'purpose' requires an understanding of intent. We need to find out, do they make conscious choices or is their motivation so instinctive that they don't understand a 'why' question at all. And the biggest of all, we need to have enough vocabulary with them that we understand their answer. ${ }^{2}$

E finalmente, num diálogo com seu colaborador físico (que retomarei mais adiante):

Louise -I know what it is.

Ian -What?

Louise -It's not a weapon, it's a gift.

${ }^{1}$ Em entrevista ao escritor Chiang, disponível no iTunes extras, este comenta: "I wanted to, uh, write a story about the step that, in a lot of Science fiction stories, they always skip over. About the process of learning an alien language, a language which has no basis in any human language, bears no similarity to any known human language and for which you don't have any translator available, there's no one who has already learned your language and their language and can act as an intermediary."

${ }^{2}$ Essa cena remete ao conhecido papel dos intérpretes nas viagens pelo Novo Mundo. No Brasil do século XVI havia a figura do língua, homem que aprendia o idioma do índio, muitas vezes de maneira forçada. O contrário também acontecia, isto é, índios eram levados à Europa para aprender o idioma do colonizador. Estes eram conhecidos como filhados. Quando colonos permaneciam em terra para aprender o idioma indígena e ajudar na colonização, eram chamados de lançados. (Reis e Bagno 86-7). 
-The weapon is their language. They gave it all to us. Do you understand what that means?

Coronel -So we can learn Heptapod, if we survive.

Louise -If you learn it, when you really learn it, you begin to perceive time the way that they do. So, you can see what's to come.

Nos dias atuais, o volume editorial de livros de viagem, inéditos ou não, originais ou adaptados, literatura de ficção, guias, antologias, biografias romanceadas ou factuais de viajantes, sites dedicados a literatura de viagem e viajantes como o Curious Travellers elaborado sob a direção de Mary-Ann Constantine ou o Women's travel writing, 1780-1800: A bio-bibliographical database criado em 2014 por Benjamin Colbert da University of Wolverhampton, além de muitos blogs, como o Mujeres viajeras ${ }^{3}$ criado em 2011 por Carla Ulloa Inostroza e inúmeros filmes, como The Arrival ${ }^{4}$ mencionado acima, são reflexo desse interesse pelo relato de viagem, pelo exótico e pelo diferente ${ }^{5}$.

${ }^{3}$ Em pesquisa feita no google, utilizando a palavra-chave blog viagem, em 0,30 segundos tem-se 163 milhões deles, principalmente dedicados a roteiros turísticos baseados nas experiências dos blogueiros, ou, como diria Michael Frayn, Travels with a typewriter.

${ }^{4}$ Alguns exemplos de filmes recentes que abordam a temática - viagem e tradução - Atlantis: the lost empire, uma produção Walt Disney Picture (2001); The Terminal, de Steven Spilberg (2004); The Interpreter de Sydney Pollack (2005) entre outros.

${ }^{5}$ Goodreaders, um catálogo aberto on-line fundado em 2006-2007 pelo engenheiro de software Otis Chandler e pela jornalista Elizabeth Kury, do Los Angeles Times; comprado pela Amazon em 2013, possui mais de 20 milhões de membros e 10 milhões de livros catalogados, mostra-nos, após rápida busca, na qual utilizamos o filtro: livros que possuam as palavras travel writing no título, 422.820 títulos ou publicações. Consultado em 15/072019. 


\section{A narrativa de viagem}

A prática da narrativa de viagem remonta os séculos e suscita, no correr do tempo, o interesse de inúmeros leitores. No século XVIII, viagem significa tanto o deslocamento quanto a narrativa que dela é feita, sendo esta apresentada como um relato do que se pretende real. A jornada apresenta-se como um apelo à reflexão histórica, distanciada, portanto, do pitoresco e da emoção.

No século XIX, os românticos utilizam essa temática em suas criações poéticas e romanescas. Se o parentesco entre a viagem e a escrita, segundo Michel Butor (04), sempre existiu, foi na época romântica que ela melhor se manifestou, principalmente na França e na Alemanha. Desse modo, devemos aos escritores da época romântica a noção de que a arte de viajar é também uma arte de escrever. Passa a vigorar, neste momento, uma forma mais livre, mais direta, mais próxima da confissão, ainda que o espírito crítico, característico do século das Luzes perpetuado no século XIX, não deixe de estar presente, bem como o testemunho histórico (Le Iraqi 44-5). A viagem transforma-se numa espécie de tema literário.

François Laplantine, em "L'ethnologie, le traducteur et l'écrivain", nos dá exemplo deste acontecimento ao destacar a transformação da Conquista da América em tema literário:

L'observation de ce qui jamais n'avait été vu est une telle aventure qu'elle transforme la Conquête en événement littéraire: écrire ce qui jamais n'avait été écrit. C'est le choc de l'Amérique, devenue passion américaine, qui les a conduits à inventer des formes nouvelles d'expression. (503)

Paralelamente, há a narrativa naturalista baseada nas instruções institucionais como museus, que explicam o que deve ser visto e de que maneira; que explicam como o viajante, por mais ordinário que seja, pode contribuir para enriquecer as coleções de plantas, insetos etc. dos museus do velho mundo se souber o quê e como 
coletar e conversar o material. Um exemplo dessa Instrução para os viajantes e empregados das colônias sobre a maneira de colher, conservar, e remeter os objetos de história natural pode ser acessada no acervo digital da Biblioteca Nacional do Rio de Janeiro.

No que concerne à narrativa de viagem, portanto, podemos dizer que possui uma tipologia variada, uma vez que se encontra em uma zona de fronteira, aberta, polivalente, que guarda em si uma série de gêneros tais como: diário íntimo, autobiografia, discurso epistolar, ensaio etnográfico, literário e científico. Esta volatilidade garante-lhe certa liberdade formal que lhe permite adaptar-se melhor às diferentes mudanças estéticas e ideológicas que afetam uma sociedade (Le Huenen 7-9).

Trata-se de um gênero flexível que parte do princípio da reescrita, da reformulação de uma experiência individual que, a partir do momento que é publicada, ultrapassa o domínio privado integrando o público. Desde o início da narrativa o escritor-viajante assina um contrato de cumplicidade com o leitor. “... one of the primary tasks of the travel writers is to demonstrate how much translation has to be done, if only to justify their narrative role as interpreter of the scenes they witness". (Cronin 36)

Segundo uma abordagem elaborada por James Clifford, retomada por Pym (2010), a viagem torna-se o principal meio de contato entre culturas, configurando os espaços nos quais a tradução cultural acontece. De acordo com Mary Louise Pratt (27), pode-se dizer que esses espaços são as "zonas de contato", i.e., o espaço social onde as distintas culturas encontram-se, chocam-se, misturam-se, quase sempre, numa relação assimétrica de dominação e subordinação.

De acordo com Michael Cronin, é necessário saber que o viajante é aquele que deixa sua "casa", mas que sempre retorna a ela, caso contrário não se trataria mais de uma viagem e sim de um exílio. O viajante deve tratar com outras línguas, diferentes da sua, e isso tem implicações sobre a capacidade de interpretação da realidade que lhe é estrangeira.

Cronin em sua obra Across the lines (02) tenta demonstrar ao leitor a função chave das questões da linguagem e da tradução du- 
rante a viagem, sublinhando o papel central do tradutor enquanto mediador cultural. Ele demonstra o papel da linguagem na construção da identidade do viajante e também da identidade do Outro. Essa construção se faz possível por propor o encontro com o outro, transformando-o e transformando a si ao mesmo tempo (Ferreira, Tradução etnográfica 65$)$.

\section{Os estudos acadêmicos}

Na obra Exploring translation theories, Anthony Pym (154) afirma que a maneira de representar as culturas ${ }^{6}$ pelo viés da viagem e dos viajantes ainda é um tema de pesquisa pouco explorado no âmbito universitário.

De acordo com Stella Franco (14-15), no meio acadêmico, os estudos da temática viagens e relatos poderiam ser divididos, sob risco de simplificação, em duas vertentes interpretativas, uma ligada à História Social, que utiliza os relatos enquanto fonte, e, a outra, à Crítica Literária, que se preocupa mais com a dimensão discursiva deles. Eu incluiria nesse rol uma terceira vertente, a dos Estudos da Tradução ${ }^{7}$, pois, assim como Ted Chiang percebeu a falta de interesse pela linguagem, pela comunicação dos humanos com os aliens nos filmes e literatura de ficção científica ( $c f$. nota 1 , acima), àqueles que se consagram aos estudos daquilo que vou chamar de tradução cultural/etnográfica no Brasil ainda se importam pouco com o assunto ${ }^{8}$.

${ }^{6}$ Segundo Laplantine, em seu artigo já citado (497), cabe à Antropologia tornar familiar aquilo que nos é estranho e estrangeiro (a cultura do outro) e tornar estranho ou estrangeiro aquilo que nos é familiar. E cabe a Etnografia a escrita da cultura. ${ }^{7}$ Área delimitada inicialmente pelo Grupo de Trabalho da Associação Nacional de Pós-graduação em Letras e Linguística - ANPOLL em 1986. A designação veio num crescendo, passando por Estudos em Tradução (entre outras), até se chegar na denominação atual de Estudos da Tradução.

${ }^{8}$ No que tange as teorias da tradução, Molína, em sua tese de doutorado, faz uma síntese das teorias tradutológicas com o intuito de verificar a partir de quando 
No entanto, esse aspecto da viagem - relação entre viajante, linguagem/cultura e escrita - tem sido tratado com indiferença pelos estudiosos e críticos causando uma série de mal-entendidos tanto no que diz respeito à experiência da viagem quanto à construção da narrativa desta. Pode-se cair, neste âmbito, em ciladas como: o erro potencial da tradução; a perda de sentido/significado; o mito da transparência; a relação entre linguagem e poder e a representação baseada em "conceitos universais". Como diria o Coronel Weber (interpretado por Forest Whitaker) em The Arrival: Será que os alienígenas/estrangeiros vieram para trazer guerra ou paz?

$\mathrm{Na}$ época atual, na qual o processo de internacionalização ${ }^{9}$ das Universidades brasileiras tem sido priorizado pelo governo, por editais de instituições de fomento e por editais internos às próprias Instituições de Ensino Superior, a necessidade de relacionar-se com o outro, com o estrangeiro, a maneira de ultrapassar/vencer as barreiras linguísticas e culturais que os separam são essenciais aos processos de mobilidade visados. O movimento de pessoas ao redor do mundo, hoje, acontece entre falantes de 7097 idiomas diferentes ${ }^{10}$ ! "The neglect is all the more telling in that one of the most commonplace experiences of the traveler is the sudden humiliation of language loss as things go disastrously wrong and familiar words reveal themselves to be worse than useless." (Cronin 02).

A afirmação de Cronin pode ser exemplificada com um pequeno trecho do romance, que será abordado mais adiante, de Daniel Grenier “... Albert claquait des doigts, à la recherche du mot juste

estas começaram a levar em conta o elemento cultural, dado que nos interessa particularmente. O primeiro teórico mencionado pela autora é Nida, com a publicação do artigo "Linguistics and Ethnology in Translation Problems", em 1945.

9 Sobre os problemas linguísticos nos processos de internacionalização, conferir notícia publicada no Independent, sob autoria de Jon Stone, em 19 de julho de 2018. Nesta, o jornalista questiona a tradução, para a língua alemã, do documento elaborado por Theresa May a respeito do Brexit. Segundo o autor, o texto era "awful to read" e "not German".

${ }^{10} \mathrm{O}$ Summer Institut of Linguistics. Language of the world, dirigido atualmente por Michel Kenmogne, foi criado em 1919 por William Cameron Townsend. 
ou de l'expression correcte, ... 'Voyons, câlisse, c'est quoi déjà? Comment qu'on dit ça déjà'..." (46).

Alguns estudos conhecidos nesse ramo do conhecimento são: Across the lines: Travel, Language and Translation, de Michael Cronin; Translating travel: Contemporary Italian Travel Writing in English Translation, de Loredana Pollezzi; Travel in Twentieth-Century French and Francophone Cultures; a coleção organizada por Carmine G. Di Biase chamada Travel and Translation in the Early Modern Period; "La traductologie, l'ethnographie et la production des connaissances", de Hélène Buzelin. No Brasil, Cristina Carneiro Rodrigues dedicou-se ao estudo da relação entre tradução, viagem e poder; Maria Elizabeth Mello vem empenhando-se na elaboração de antologias de textos de viajantes, como por exemplo, O Brasil do século XIX, no olhar de Charles Expilly; Eduardo Luís A. Batista defendeu uma tese (IEL-UNICAMP) intitulada: Poética da representação cultural: relações entre viagem e tradução na Literatura Brasileira; e Alice M. Araújo Ferreira vem trabalhando, mais especificamente, com tradução etnográfica.

\section{A estrada de tijolos amarelos}

A proposta de análise pretendida parte do paradigma da descrição na tradução etnográfica enquanto realidade social apreendida a partir do "ver", da realidade social transformada em linguagem, do esforço de transformar o olhar em escrita, segundo nos explica Laplantine, em La description etnographique, e de sua relação com a tradução, como vem pesquisando Maria Alice Ferreira.

Em seguida, como instrumentos de análise da descrição na tradução etnográfica, utiliza-se o modelo de Romàn Jakobson (6465), segundo o qual há três espécies de tradução:

1. A tradução intralingual ou reformulação (rewording) consiste na interpretação dos signos verbais por meio de outros 
signos da mesma língua. Representada, no presente artigo, pela literatura de ficção de Christelle Dabos.

2. A tradução interlingual ou tradução propriamente dita consiste na interpretação dos signos verbais por meio de alguma outra língua. Representada aqui pelo relato de Adèle Toussaint-Samson e pelo romance de Daniel Grenier.

3. A tradução inter-semiótica ou transmutação consiste na interpretação dos signos verbais por meio de sistemas de signos não verbais. Representada aqui pelo filme The Arrival e pelo conto de Chiang que lhe deu origem; na tradução da linguagem escrita à linguagem midiática (filme); no contato entre a linguista e os alienígenas e naquilo que este lhe proporcionou, a saber, uma nova maneira de pensar diretamente ligada à concepção diferente do tempo.

Além do conceito de Cultural-Specif Items/Itens culturalmente marcados (ICE), desenvolvido por Javier Franco, que diz respeito a um conjunto de palavras, expressões específicas de uma determinada cultura de partida que, uma vez colocada em relação com a cultura de chegada não têm "equivalentes", o que provoca aquilo que o autor chama de "intercultural gap", responsável por levar a tradução para além do campo linguístico. "The main difficulty with the definition lies, of course, in the fact that in a language everything is culturally produced, beginning with language itself" (57). A presença dos ICEs pode, no entanto, demonstrar certas características da sociedade, da cultura e do país, e são um aspecto bastante usual nas narrativas de viagem sobre o Brasil de meados do século XIX e início do XX (Camargo 163) e essenciais para se atingir os objetivos ora propostos.

Assim, palavras funcionam como sinais de intraduzibilidade, mas num espaço de tradução. A realidade que está acontecendo em uma língua estrangeira está sendo transmitida ao leitor na linguagem da narrativa de viagem, no entanto, há sempre a presença de termos exóticos que podem aparecer no relato de maneira discreta ou destacada/sublinhada. "The reader is translated into a foreign climate through the untranslated" (41). 
Ainda segundo Michael Cronin (92), "Travel, in its most progressive form, carries the ethical project of translation to the cultural and spiritual level as it seeks to engage fully with other cultures". Maria Alice Ferreira salienta o processo de desterritorialização "um estranhar de nós-mesmos [que] permite a percepção que o que tínhamos como 'natural' na nossa sociedade, em particular a língua que falamos ... é, na realidade, um fato social como já dizia Saussure" (Tradução etnográfica 67).

\section{Labirinto de espelhos}

Como disse o etnólogo francês Laplantine (L'ethnologue 502) ao escrever sobre sua experiência na América Latina, ou Cronin ao analisar relatos ficcionais e reais de viagens a países de mesma língua materna, por mais que pareçamos iguais há sempre algo que deixa tudo diferente: a mudança de cenário, o sotaque, as expressões regionais, etc.

Christelle Dabos, ao escrever Le passe-miroir cria um universo imaginário incrível. A história se passa em um mundo esfacelado, onde cada um dos pedaços restantes constituiu-se em um arco governado por um espírito familiar imortal. Ophélie, a protagonista, nasceu em Anima, um pequeno arco onde os animistas têm o poder de dar vida aos objetos. Após recusar dois pedidos de casamento, Ophélie é prometida a um estrangeiro, originário do Pólo, arco governado pelo senhor Farouk. Por tratar-se desta vez de um casamento diplomático, não há escapatória possível. Ela muda-se, assim, para o Polo. Os hábitos, os costumes e o sotaque, um tanto gutural, dos que aí vivem, causam-lhe estranheza e dificuldade de comunicação. A certa altura da narrativa, Ophéfie decide ir à Babel, arco governado pelos espíritos gêmeos Pollux e Hélène, procurar por seu marido desaparecido. Neste local ela encontra a diferença, mas não só: primeiro há a constatação da grande variedade de tipos e de sotaques: 
... Là, assise sur la bordure de la fontaine [...], elle prit un moment pour observer attentivement le marché. La diversité des peaux, des morphologies et des accents était celle d'une population cosmopolite: il n'y avait pas ici une, mais plusieurs familles. Pourtant, ils semblaient tous ne former qu'un seul peuple où Ophélie tenait le rôle d'intruse. (s/p)

Em seguida, há um mal-entendido cultural cuja base está em um rígido código de vestimenta:

- Ce que vous devez comprendre, Miss Eulalie [nome adotado pela personagem Ophélie], c'est qu'ici les gens sont exactement ce à quoi ils ressemblent. Nous avons, au même titre qu'un code civil et un code pénal, un code vestimentaire très strict. Mon père et moi, par exemple, sommes tenus par la loi de porter du blanc. C'est la non-couleur des sans-pouvoir. ...

... Vous êtes menue, mais je ne suis pas très grand non plus. Mes vêtements seront presque à votre taille.

- Parce qu'il sera moins choquant pour moi de porter des habits d'homme? ... Nous ne faisons pas de différence entre les sexes ici. J'en déduis que chez vous les hommes ne portent pas de tenues comme la vôtre? ... Non, effectivement. - C'est intéressant. Cela étant, Miss Eulalie, le principal problème avec votre robe est que son modèle ne figure pas dans notre code vestimentaire. Ne pas respecter ce code en public est interprété comme un acte de provocation. Ce qui est évidemment très mal perçu. ...

- La composition vestimentaire varie selon l'âge, la profession et l'état civil... (s/p, grifo meu)

E, por fim, após conviver com pessoas de culturas diferentes por muito tempo, ela acaba por questionar se Ophélie não havia se transformado em Eulalie, a identidade falsa que havia adotado 
para procurar seu marido. Tal fragmento leva-nos à epígrafe deste artigo que diz: "Il faut tâcher de pénétrer ce qu'ils pensent, et non prétendre les faire penser à notre manière" (Gerondo apud Laplantine, L'éthnologue 499).

... Cette imposture était plus conséquente que toutes les comédies qu'elle avait joué par le passé. Ce n'était pas seulement un déguisement, comme l'avait été la livrée de Mime [outro disfarce de Ophélie]: c'était une autre peau qui, jour après jour, était devenue une seconde nature. À force de se prendre pour Eulalie, pouvait-elle toujours prétendre être Ophélie? (s/p, grifo теи)

Daniel Grenier em L'année la plus longue, romance que, tendo como pano de fundo a história dos Estados Unidos e Canadá, conta a saga da família Langlois por meio de viagens do patriarca e de seus ancestrais, em certo momento da narrativa descreve a sensação de frustração do filho Thomas, franco-anglófono, ao escutar seu pai, Albert ${ }^{11}$ :

... dans la maison on prononçait son nom d'une façon étrangère: To-ma. Son père le prononçait comme ça. Il savait que son père était un homme qui ne parlait pas la même langue que lui et que tout le monde autor, et que c'était insolite et merveilleux. À l'intérieur de la maison il était To-ma et à l'extérieur il était Thaw-muss, avec un $s$ final bien clair. Son $s$ disparessait quand il passait le seuil de la porte... (25, grifo теи)

Parfois il [Thomas] s'arrêtait d'écouter ce que son père [francófono] disait pour se concentrer sur la différence entre les mots que ce dernier prononçait et ceux qu'il

${ }^{11}$ Albert representa o viajante, o estrangeiro. 
entendait ailleurs. Comment pouvaient-ils être les mêmes mots, si leur son était si différent? Il devait se faire violence pour arrêter de penser à leur son et retrouver leur sens, ce qu'ils cherchaient à exprimer, par la bouche de son père. (27, grifo теu)

Ou ainda, quando Albert, recém-chegado à Chattanooga (Tennessee), vai a um restaurante:

- Now, that's a lot of sugar.

- Hm? Oh. Yes. I like it, hm, really sucré, like that.

Son accent était si prononcé qu'elle [a garçonete] a fait semblant de comprendre en souriant jusqu'à ce qu'elle comprenne quelques minutes plus tard, en analysant les sons, en les décorticant... (41)

Ainda de acordo com o etnólogo, o universo não fala. As coisas passam a existir por meio das palavras, palavras que respondem a outras palavras, e então "l'univers devient bourdonnant de consonnes et de voyelles. ... Nous naissons et grandissons à l'intérieur du langage ou plutôt d'une langue particulière, une langue qui nous comprend autant que nous la comprenons" (L'éthnologue 499). Thomas e Albert, citados acima, mostram-nos o quanto esta afirmação é pertinente.

Em meados do século XIX, uma francesa, Adèle ToussaintSamson, casada com um francês nascido em terras Tupiniquins, toma residência no Brasil por cerca de dez anos (1850-1862) ${ }^{12}$. Ao retornar a seu país natal, escreve uma pequena narrativa - Une parisienne au Brésil -, descrevendo aquilo que vivenciou enquanto esteve nos trópicos. Para fazer-se entender por seu público leitor francês, uma vez que lhe faltam palavras que descrevessem a reali-

12 As idas e vindas de Adèle Toussaint à França acontecem, mas não são devidamente descritas em seu relato. 
dade brasileira, a escritora-viajante fez uso de termos e expressões em português seguido: ora da tradução literal, ora de longas descrições, ora de notas, ora de imagens ${ }^{13}$ em seu processo tradutório.

A figura de Adèle é curiosa, pois ela foi, além de escritora ${ }^{14}$, poeta, tradutora. Este fato faz com que seja bastante cuidadosa, que reflita sobre os termos que utilizará para descrever aquilo que vê, como demonstra os trechos abaixo:

Coitadinho d'elle ! Ce coitado ! est intraduisible. Il veut dire : Pauvre malheureux ! Mais c'est plein d'une compassion mêlée de mépris, que nous ne pouvons rendre en français." (178)

C'est ce qui fait que j'ai, depuis, toujours saudade (1), comme disent les Brésiliens, de l'Amérique du Sud, et que je voudrais la revoir encore une fois avant de mourir.

Souvenir mêle de regrets, mot charmant, intraduisible. (219)

Já quase chegando ao final de seu relato, de volta à sua terra natal, a autora-viajante quase se lamentar da civilização. Da janela de um trem, observa o quadriculado colorido de terrenos cultivados e seus pensamentos a levam à natureza brasileira, sua vastidão, sua riqueza e exuberância e sente saudades. Adèle já não era mais a mesma.

${ }^{13}$ Um exemplo é a foto "Negrèsse au marche" (Toussaint-Samson 40-1) que traz aos olhos do leitor o retrato mais "fiel" possível da realidade social da negra Mina com seu tabuleiro de frutas para vender no mercado.

${ }^{14}$ Em 1870 Adèle traz à lume seu primeiro livro intitulado Épaves, sourires et larmes. Dez anos mais tarde é a vez de Les chemins de la vie, que lhe trouxe prêmio da Academia Francesa. Em 1883 lança Une pariesienne au Brésil e, por fim, La comtesse Diane (uma comédia) e L'histoire de Messire Jahan et de Ursule dame. Em anexo ao pequeno relato sobre o Brasil, a autora insere traduções de textos de Magalhães e Gonçalves Dias. 
Pourtant, bien des étonnements et bien des désillusions m'attendaient au retour. Mon pays, qui était resté si beau dans mon souvenir, me sembla stérile, triste, gris, auprès de celui que je venais de quitter. ... (214) ...

Sous notre mesquine civilisation, j'avais bien de la peine à retrouver la nature, de même que j'ai bien de fois cherché le ciel, que les hautes Maison de nos villes dérobent à nos regards. (216, grifo meu)

\section{À guisa de conclusão}

Como já disse Laplantine (499), o universo não fala; tudo vem ao mundo por meio de palavras; dessa forma, a proposta de análise que procurei apresentar neste artigo buscou demonstrar o papel da linguagem/cultura na construção da(s) identidade(s) ${ }^{15}$ de um autorviajante e, também, do outro. Tal fato pode ser verificado na figura da linguista do filme The Arrival, que vê sua vida transformar-se ao compreender a arma/presente dos alienígenas; na protagonista, Ophélie, criada por Christelle Dabos, que questiona sua própria identidade e no pequeno relato Une parisienne au Brésil, de Adèle ToussantSamson, no qual a viajante se deixa levar pela saudades e mostra ao leitor como sua opinião sobre a "civilização" havia mudado.

Dito isto, verifica-se que tal proposta pode ser aplicada a um corpus variado: relato de viagem, literatura de ficção, romance histórico e filme. Seria reflexo da maleabilidade do gênero literatura de viagem? Espero que tal proposta tenha mostrado sua eficácia em identificar as influências na construção da(s) identidade(s) do viajante e de seu "semelhante" estrangeiro.

${ }^{15}$ Vale lembrar, juntamente com Alice Ferreira e Laplantine, que a identidade não é inalterável.

Cad. Trad., Florianópolis, v. 41, $\mathrm{n}^{0}$ 1, p. 46-65, jan-abr, 2021. 


\section{Referências}

Batista, Eduardo Luis Araújo de Oliveira. Poética da representação cultural: relações entre viagem e tradução na literatura brasileira. Tese do Instituto de Estudos da Linguagem. Campinas: UNICAMP. 2010, 355p. Disponível em: $\quad$ http://repositorio.unicamp.br/bitstream/REPOSIP/270248/1/Batista EduardoLuisAraujodeOliveira_D.pdf. Acesso em: 08/07/2020.

Butor, Michel. "Le voyage et récriture". Romantisme. 4, (1972): 4-19. Portal Persée. 15 out 2019. < https://www.persee.fr/doc/roman_0048-8593_1972_ num_2_4_5399>.

Buzelin, Hélène. "La traductologie, l'ethnographie et la production des connaissances.". Meta: journal des traducteurs/Meta: Translators' Journal. 49.4, (2004): 729-746. 15 mar 2020. https://www.erudit.org/en/journals/meta/1900v1-n1-meta832/009778ar.pdf.

Camargo, Katia A. Franco. "Relatos de viagem e a tradução de palavras culturalmente marcadas: um estudo de caso". Cadernos de Tradução. 37. 2, (2017): 159-176. Portal de periódicos da UFSC. 17 out. 2019. < https://periodicos.ufsc. br/index.php/traducao/article/view/2175-7968.2017v37n2p159/34073>.

Chandler, Otis; Kuray. Goodreaders. Web. 2020. https://www.goodreads.com.

Chiang, Ted. Stories of Your Life and Others. New York: Vintage Books, 2016.

Colbert, Benjamin. Women's travel writing, 1780-1800: A bio-bibliographical database. Web. 2014. http://www4.wlv.ac.uk/btw/about.

Constantine, Mary-Ann; Leask, Nigel. Curious Travellers. Web. 2014. https:// curioustravellers.ac.uk/en/.

Cronin, Michael. Across the Lines: Travel, Language, Translation. Irlanda: Cork University Press, 2000. 
Dabos, Christelle. La Passe-miroir: la mémoire de Babel. Paris: Gallimaard Jeunesse, 2019.

El Iraqi, Mohammed Amine. "Récit littéraire et récit journalistique dans la littérature de voyage: réflexion sur les écrits de F. R. Chateaubriand". Revue Langues, Cultures et Sociétés. 4.1, (2018): 44-57. 21 set. 2019. < https://revues. imist.ma/indexp? journal $=$ LCS\&page $=$ article \&op $=$ view \&path $\% 5 B \% 5 D=1239$ $0 \&$ path $\% 5 \mathrm{~B} \% 5 \mathrm{D}=7150>$.

Ferreira, Alice. M. "Tradução etnográfica - poética do encontro". Crítica $e$ Tradução do Exílio: Ensaios e Experiências, Ferreira, Alice M. A., et al. (Orgs). Goiânia: Editora da Imprensa Universitária UFG, 2017, pp. 55-91.

Forsdick, Charles. Travel in Twentieth-Century French and Francophone Cultures: The Persistence of Diversity. Oxford: Oxford University Press, USA, 2005.

Franco, Javier Aixelá. "Cultural-Specif Items in translation”. Translation, Power and Subversion, Román Alvarés Rodríguez e Carmen-Africa Vidal (Orgs.). Philadelphia: Multilingual Matters LTD, 1996, pp. 52-78.

Franco, Stella M. S. Viagens e Relatos. Representações e Materialidade nos Périplos de Latino-Americanos pela Europa e pelos Estados Unidos no século XIX. São Paulo: Intermeios, 2018.

Frayn, Michael. Travels with a Typewriter: A Reporter at Large. Londres: Faber \& Faber, 2011.

Grenier, Daniel. L'année la plus longue. Montréal: Le Quartanier, 2017.

INSTRUÇÃO para os viajantes e empregados das colônias sobre a maneira de colher, conservar, e remeter os objetos de história natural. Arranjada pela administração do Museu de História Natural de Paris. Traduzida por ordem de Sua Majestade Fidelíssima, expedida pelo Excelentíssimo ministro e secretário de Estado dos negócios do Reino. Do original francês de 1818. Rio de Janeiro, Imprensa Régia, 1819. 19 set. 2019. < http://objdigital.bn.br/acervo_digital/ div_obrasraras/or84809/or84809.pdf > 
Jakobson, Román. Linguística e Comunicação. Tradução de Izidoro Blikstein e José Paulo Paes. São Paulo: Cultrix, 2011.

Kurtti, Jeff. “Atlantis the Lost Empire: The Illustrated Script.” (2001).

Laplantine, François. “L'ethnologue, le traducteur et l'écrivain”. Meta. 40.3, (1995): 497-507. Base Érudit. 15 out. 2019. < https://www.erudit.org/fr/revues/ meta/1995-v40-n3-meta182/003398ar/>.

Laplantine, François. La Description Ethnographique. Paris: Armand Colin, 1996.

Le Huenen, Roland. “Le récit de voyage: l'entrée en littérature”. Études Littéraires. 20.1, (1987): 45-61. Base Érudit. 15 out. 2019. <https://www. erudit.org/fr/revues/etudlitt/1987-v20-n1-etudlitt2233/500787ar/ > .

Mello, Maria Elizabeth Chaves de. Lições de crítica: conceitos europeus, crítica literária e literatura crítica no Brasil do século XIX. Vol. 4. Rio de Janeiro: Editora da Universidade Federal Fluminense, 1997.

Molina, Lucía. “Análisis Descriptivo de la Traducción de los Culturemas ÁrabeEspañol”. Tese de doutorado, Universidade Autônoma de Barcelona, 2001.

Nida, Eugene. "Linguistics and ethnology in translation-problems.". Word 1.2, (1945): 194-208. Disponível em: https://www.tandfonline.com/doi/pdf/10.1080/ 00437956.1945.11659254. Acesso em: 15/03/2020.

Pratt, Mary Louise. Os Olhos do Império. Relatos de Viagem e Transculturação. Bauru: EDUSC, 1999.

Pollack, Sydney, et al. The interpreter. Working Title Films, 2005.

Polezzi, Loredana. Translating Travel: Contemporary Italian Travel Writing in English Translation. Amsterdã: Routledge, 2017.

Pym, Anthony. Exploring Translation Theories. Londres: Routledge, 2010. 
Reis, Dennys S. e Marcos BAGNO. "Os intérpretes e a formação do Brasil: os quatro primeiros séculos de uma história esquecida". Cadernos de Tradução. 36.3, (2016): 81-108. Base Scielo. 17 out. 2019. <http://www.scielo.br/scielo. php?pid $=$ S2175-79682016000300081\&script $=$ sci_abstract\&tlng $=$ pt $>$.

Spielberg, Steven; Hanks, Tom; Zeta-Jones, Catherine. The terminal. USA: DreamWorks, 2004.

Summer Institut of Linguistics. "Language of the world". Web. 2018. https:// www.sil.org/worldwide.

The Arrival. Dir. Denis Villeneuve. Atores Amy Adams, Jeremy Renner, Forest Whitaker, Rot. Eric Heisserer. Studio 21 Laps Entertainment, 2016.

Toussaint-Samson, Adèle. Une Parisienne au Brésil. Paris: Paul Ollendorf, 1883. 10 jul. 2018. < https://gallica.bnf.fr/ark:/12148/bpt6k5747774p.texteImage>.

Toussaint-Samson, Madame. “A ma sœur”. Épaves, sourires et larmes: poésies. Paris: E. Dentu Editeur, 1870, pp.101-108. BNF, Gallica. Disponível em: http:// gallica.bnf.fr/ark:/12148/bpt6k57208000/f59.image. Acesso: 15/11/2019

Ulloa, Carla. Mujeres Viajeras. Web. 2007. https://www.mujeresviajeras.com.

Recebido em: $17 / 07 / 2020$

Aceito em: 21/10/2020

Publicado em janeiro de 2021

Katia Aily Franco de Camargo. E-mail: kafcamargo@gmail.com. Orcid : http:// orcid.org/0000-0001-6463-8976. 